\title{
Experimental Assessment of the Effect of PVC Attachments as Repair of RC Beams
}

\author{
David Naish", Justin Nguyen, Oscar Jimenez, Brake Hoenisch \\ Department of Civil and Environmental Engineering, California State University, Fullerton, CA, 92832, USA \\ *Corresponding Author: dnaish@fullerton.edu
}

Copyright (C) 2013 Horizon Research Publishing All rights reserved.

\begin{abstract}
The purpose of this project is to test the effectiveness of using polyvinyl chloride (PVC) structural angles to increase the capacity of reinforced concrete (RC) beams. Two $1 / 2$-scale beams with different reinforcement configurations were loaded to approximately twice the yield displacement, unloaded, and subsequently repaired and reloaded to failure. Results indicate that the PVC attachments increased the capacity of the beams by $10-15 \%$. While the increase in strength was modest, it may be sufficient for cases in which only minor improvement is necessary and thus does not justify the high cost of other repair techniques.
\end{abstract}

Keywords Reinforced Concrete, Beam, Repair, Testing

\section{Introduction}

Reinforced concrete beams are commonly used in building applications due to their high strength and relatively low cost. During an earthquake, a building's primary lateral force-resisting system is expected to take the loading and sustain major inelastic deformations. However, the non-participating (gravity load-resisting) system must also sustain these inelastic deformations to maintain compatibility with the rest of the structure. Damage to these systems, especially in older buildings, is common, as is the need for their repair and potential retrofit [1].

Minor repair techniques may be used in cases where cracks form following extreme loading. These are not usually appropriate if there is major damage to the concrete or steel reinforcement. Epoxy injection of cracks is a method used for simple repair of small cracks [2]. It involves allowing a low-viscosity epoxy resin to fill in and re-bond cracks. The procedure is slow and labor-intensive, but does provide a reasonable option for returning the concrete to its pre-cracked state [3]. Minor repair techniques such as this do not typically provide improved behavior; rather they simply attempt to bring the material to its original state.

Major repair of structural members may be required when there is substantial damage to the concrete (spalling, crushing, loss of confinement) and/or steel reinforcement (slip, pullout, buckling, fracture) following a major event. It requires substantial labor costs and generally involves removal of concrete, mechanical splicing of damaged rebar, reforming, and placement of concrete [4]. Major repair techniques such as this also do not typically aim to improve behavior (although they may if so desired).

If the members are in need of improvement prior to future extreme events, strengthening techniques can be used to improve the behavior. One common strengthening technique is the addition of interior reinforcement to help improve strength and reduce cracking at key locations. This is quite labor intensive as it involves drilling the concrete, placing reinforcement, and grouting the hole to provide bonding [4]. Exterior strengthening techniques involve the addition of new structural members (beams, columns, walls) to the system to provide support for a weak or damaged existing member. These techniques are somewhat easier to perform, but are intrusive as they take up usable space [4]. Strengthening techniques such as these are costly and used to dramatically improve the behavior of structural members that are deficient.

More recently-developed repair and strengthening techniques typically involve the use of high strength and stiffness carbon fiber reinforced polymer (CFRP) sheets that bond to the concrete using a low viscosity epoxy resin [5]. The procedure is fairly labor intensive and requires skilled workers to install. However, it is neither intrusive to the surrounding area nor invasive to the integrity of the structural member. Studies have shown that this repair technique is an excellent way to dramatically increase the elastic stiffness and strength of a concrete member, especially following minor damage $[6,7]$. Due to the high strength and stiffness of the material, delamination failure in the epoxy is typically the most common mode of failure for beams repaired with CFRP [8]. Additionally, changes to the stiffness of different elements in a structure can drastically affect the behavior of the system as a whole; even members that aren't considered part of the lateral force resisting system can be expected to cause redistribution of lateral forces if their increase in stiffness is sufficiently large [9]. 
The purpose of this study is to investigate the effectiveness of a repair system that is simpler to apply, but for cases where substantial improvements are not needed. This is often the case in older construction, whose design no longer meets the letter of the code. The only option is expensive retrofit or rehabilitation using one of the previously mentioned techniques, whereas only minor improvements are necessary to make the design strength sufficient.

\section{Materials and Methods}

\subsection{Test Specimen Geometries}

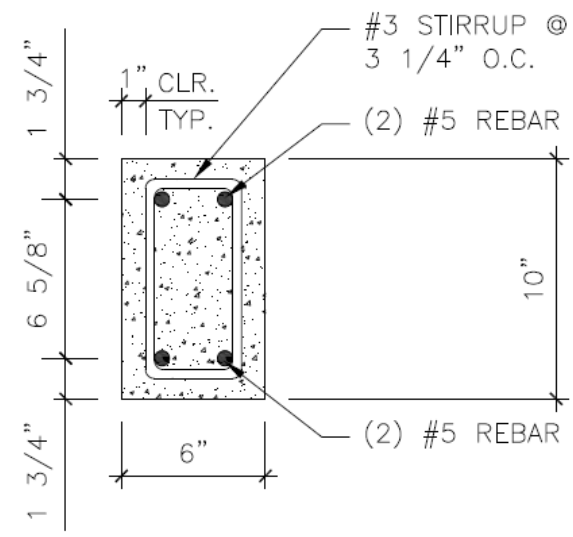

(a)

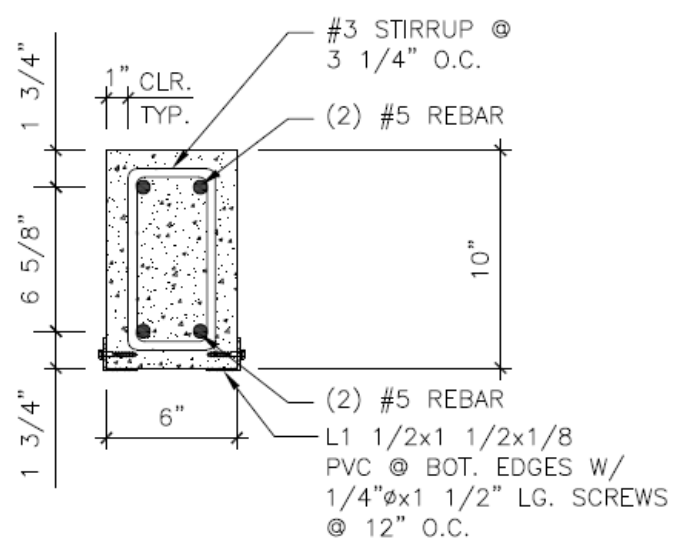

(b)

Figure 1. Cross section of (a) Beam land (b) Beam $1 \mathrm{R}$ and Beam 2.(1 in. $=25.4 \mathrm{~mm}$ )

In total, six different tests were performed on four specimens. The beam cross sections are shown in Figs. 1 and 2. All beams have the same cross section dimensions of $10^{\prime \prime} \times 6 "(254 \mathrm{~mm} \times 152.4 \mathrm{~mm})$. Beams 1 and 2 were reinforced with 2-\#5 bars on the top and bottom. Beams 3 and 4 were reinforced with $2-\# 4$ bars and 2-\#3 bars in two layers at both the top and bottom. All four beams had the same reinforcement ratio. Beams 1 and 3 were loaded beyond the yield point and were then repaired by attaching $1.5^{\prime \prime} \times 1.5^{\prime \prime} \times 0.125^{\prime \prime}(38.1 \mathrm{~mm} \times 38.1 \mathrm{~mm} \times 3.18 \mathrm{~mm}) \quad \mathrm{PVC}$ angles with concrete screws at 12 " on center. The repaired beams were then tested. The repaired specimens are indicated with an $\mathrm{R}$ after the name, Beam 1R and Beam 3R. Beams 2 and 4 were tested only after attaching the same $1.5^{\prime \prime} \times 1.5^{\prime \prime} \times 0.125^{\prime \prime}(38.1 \mathrm{~mm} \times 38.1 \mathrm{~mm} \times 3.18 \mathrm{~mm}) \mathrm{PVC}$ angles to the bottom edges of the beams. All beams were 12' (3.65 m) long.

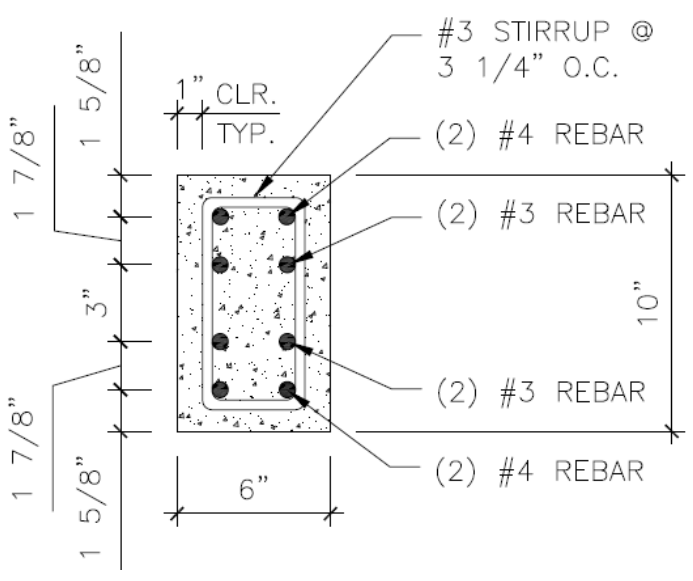

(a)

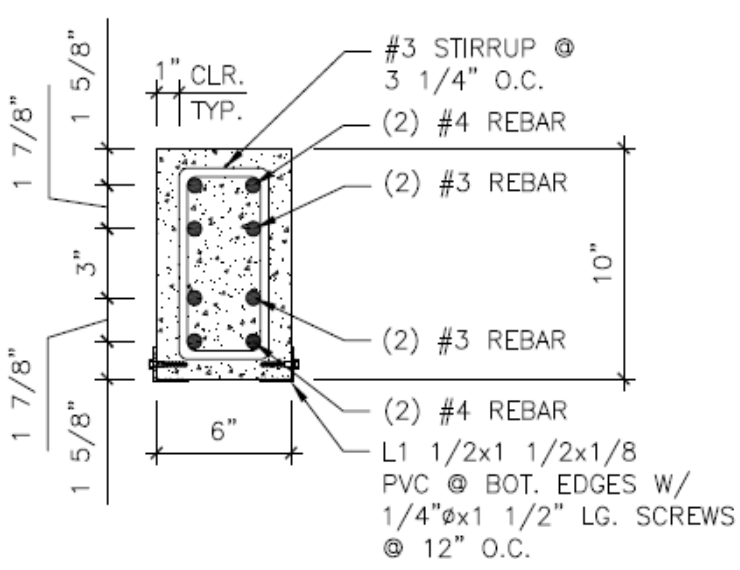

(b)

Figure 2. Cross section of (a) Beam 3 and (b) Beam 3R and Beam 4.(1 in. $=25.4 \mathrm{~mm}$ )

\subsection{Material Properties}

Concrete cylinders measuring 6" (152.4 mm) in diameter by 12 " $(304.8 \mathrm{~mm})$ in height were cast along with the beams to measure the actual concrete compressive strength. Compression tests were performed on these cylinders and the average concrete strength was measured to be 5062 psi(34.9 $\mathrm{MPa})$. Based on manufacturer specifications, the yield strength of the PVC was taken to be $12.8 \mathrm{ksi}(88.3$ $\mathrm{MPa})$ and the modulus of elasticity was assumed to be 411 $\mathrm{ksi}(2833 \mathrm{MPa})$. For calculation purposes, the concrete was assumed to follow a Hognestad stress-strain relationship, while the steel and PVC materials were assumed to follow an elastic perfectly plastic stress-strain model [10]. These 
material relations are summarized in Figure 3 below.

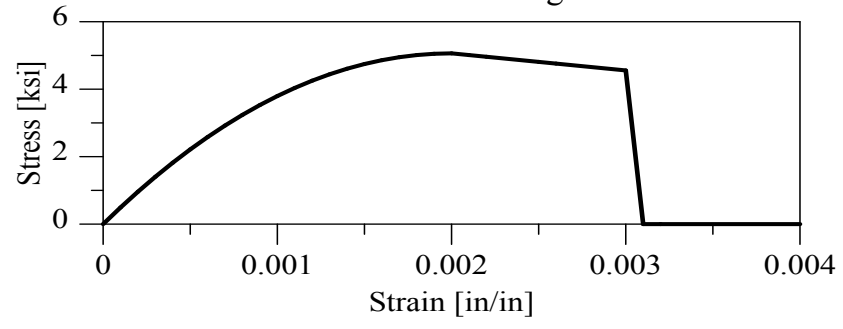

(a)

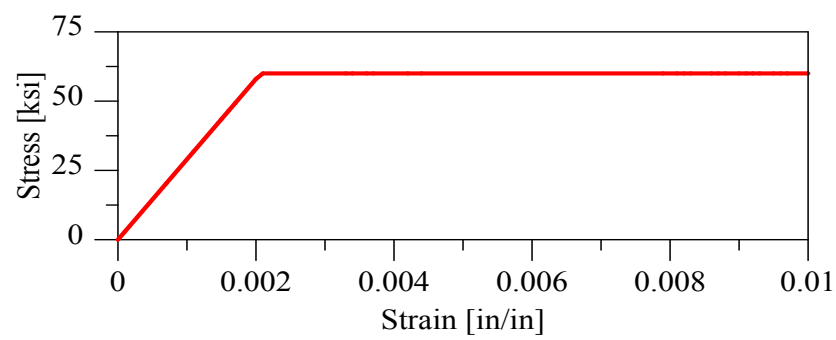

(b)

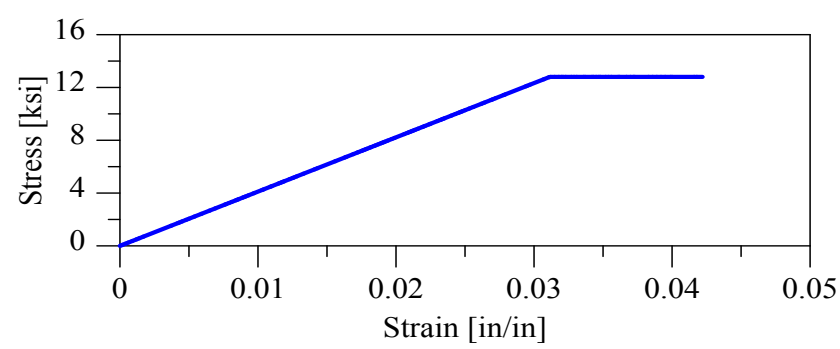

(c)

Figure 3. Stress-strain relationships for materials used in test specimens: (a) concrete, (b) steel, and (c) PVC. $(1 \mathrm{ksi}=6.89 \mathrm{MPa})$

\subsection{Test Setup and Instrumentation}

All beams were tested using the same setup. End restraints for the beam were pin supports allowing free rotation. The clear span between supports was 11'-10" (3.61 $\mathrm{m})$. Each beam was subjected to two equal point loads applied at +/- 1'-6" (+/-0.457 m) symmetrically about the centerline via a single actuator. The load was applied at a constant rate of $1000 \mathrm{lbs} / \mathrm{min}(4.45 \mathrm{kN} / \mathrm{min})$ to ensure a quasi-static load rate.

The midspan displacement was measured by one $+/-5$ " $(+/-127 \mathrm{~mm})$ wire potentiometer attached directly to the bottom of the concrete beam. The load was measured by a load cell in the actuator. The test setup and external instrumentation scheme is shown in Fig. 4.

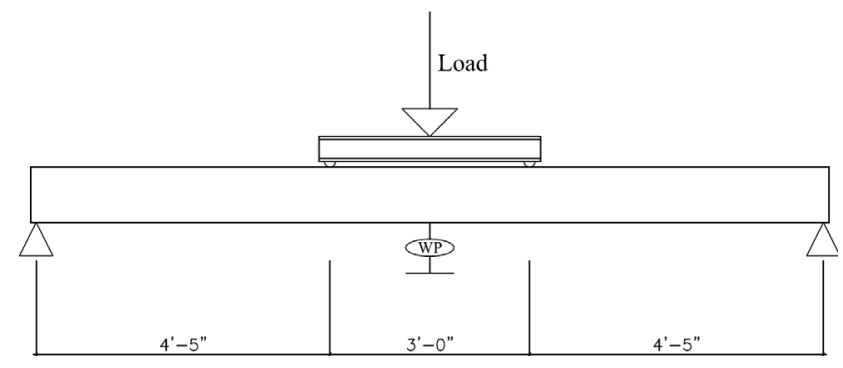

(a)

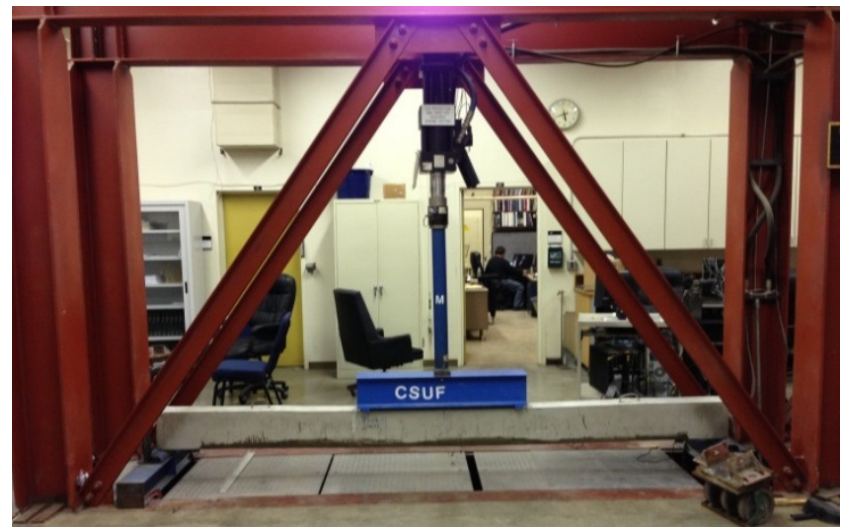

(b)

Figure 4. Experimental setup and instrumentation (a) schematic and (b) photo. $(1$ in. $=25.4 \mathrm{~mm})$

\subsection{Repair Methodology}

Beams 1 and 3 were tested beyond the yield point, unloaded, and repaired. Beams 2 and 4 were not repaired; rather they were prepared with the PVC angles prior to testing. The purpose of this was to directly gage the impact of the PVC itself, removing any effects of post-yield damage and strain hardening in the steel.

As discussed previously, many typical repair techniques involve the use of low viscosity epoxy resin in conjunction with fiber-reinforced polymer sheet or wrap. A similar approach was initially taken to repair the beams in this study with PVC angles. However, the low viscosity of the epoxy combined with the lack of flexibility in the PVC did not allow for adequate bonding with the concrete. Higher viscosity epoxy would have provided a better bond between the two materials. One problem with epoxy as a means for attachment in this specific application is the set/cure time. Typical set times range from five minutes to two hours with typical cure times ranging from one to seven days. The large set/cure time and the increased cost of the material may limit the use of high strength epoxies to situations where major repair is necessary. Additionally, special care must be provided to ensure that the epoxy is uniformly applied along the entire length of the repair region. Any gaps in the glue could present potential failure planes and lead to premature delamination.

For the purposes of this study, the PVC angles were attached with concrete screws. This was accomplished by first clamping the angles to the concrete at the bottom corners.Holes were drilled at 12 " $(304.8 \mathrm{~mm})$ on center on both the sides and bottom of the beam using a masonry drill. Finally, 1/4" (6.35 mm) diameter concrete anchors were screwed into the concrete using an electric drill. Specifically, Tapcon 0.25 " $\times 1.75 "(6.35 \mathrm{~mm} \times 44.5 \mathrm{~mm})$ blue hex-head concrete anchors were used. Note that the screws had a sealed finish to provide durability and resistance to corrosion, but were not additionally sealed or anchored in epoxy. The installation process was simple and 
cost-effective as it could be completed by one worker, or by two workers in an assembly line fashion. Due to the scale of the test specimens, it was necessary to embed the screws in the cover concrete. This is undesirable as the cover concrete does not provide substantial embedment, especially in the case of cyclic loading. That is not a major concern in the case of a gravity system however, as the screws are anchored in the tension regions of the beam. However, the presence of the screws does introduce a potential crack initiation point. An elevation view is shown in Figure 5 below.

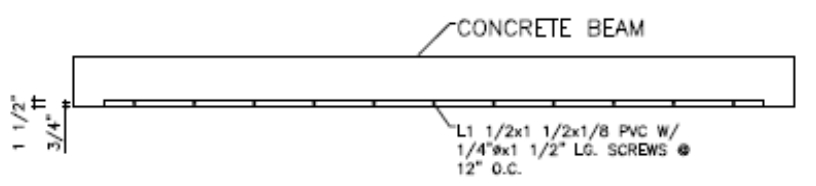

(a)

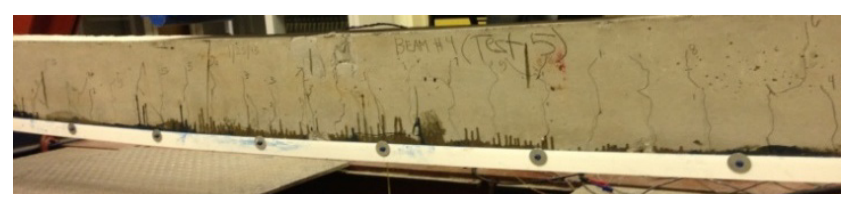

(b)

Figure 5. Repair methodology (a) schematic and (b) photo. ( 1 in. $=25.4$ $\mathrm{mm})$

\section{Results and Discussion}

Results are presented in three forms: 1) expected strength and stiffness based on moment - curvature, 2) experimentally determined strength and stiffness based on load - deformation behavior, and 3) observation of damage during testing.

A moment - curvature analysis was performed on the four beam cross sections tested using the material properties described in section 2.2. The predicted moment vs. curvature behavior of the specimens both with and without PVC are shown in Figures 6 and 7 below. These figures show the expected effect of the PVC on the behavior of the reinforced concrete beam. In both cases, because the PVC was of very low stiffness compared to the reinforced concrete, the elastic behavior of the beams was unaffected by the presence of the additional material. This is important to note as substantially changing the stiffness of the structure can change the overall behavior of a structure [9]. Specifically, changes in stiffness to certain members can alter load distribution throughout the structure. This is an important fact for gravity systems as they are typically designed to be much less stiff elastically than the lateral force resisting systems. If the gravity members are substantially stiffened, they may take more load than that for which they were originally designed.

While the relatively low stiffness of the PVC resulted in minimal impact on the elastic behavior of the beams, once those beams started to yield and deform more substantially, the PVC was able to develop more of its strength. This allowed for a nominal increase of $10-15 \%$ in strength in the post-yield range. While the expected increase was not substantial, it may be sufficient for cases where only small repair or improvement is necessary. Specifically, oftentimes a code change will result in a deficiency within a structural member. Even if the deficiency is small, retrofit or replacement of the beam may be required. In cases where the strength is deficient by only a small amount $(<20 \%)$, costly retrofit options such as those discussed in section 1 could be avoided by implementing a cheaper alternative.

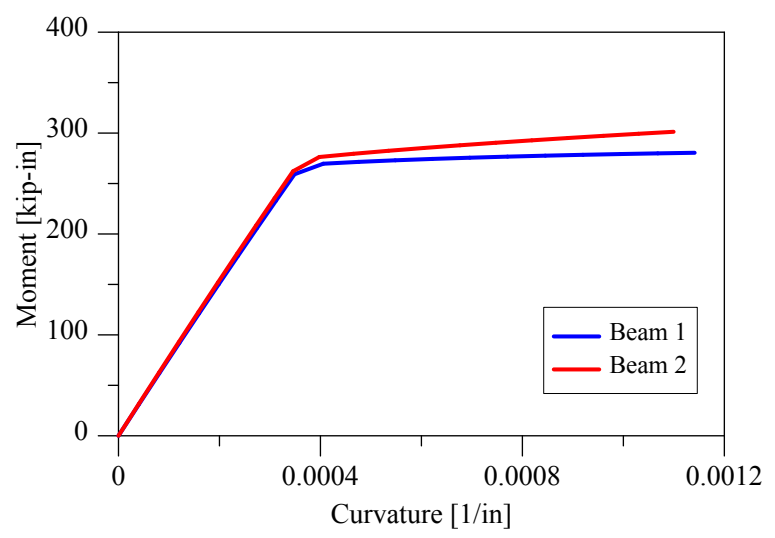

Figure 6. Moment-curvature relationship for Beams 1 and 2.(1 in. $=25.4$ $\mathrm{mm}, 1$ kip. $=4.45 \mathrm{kN}$ )

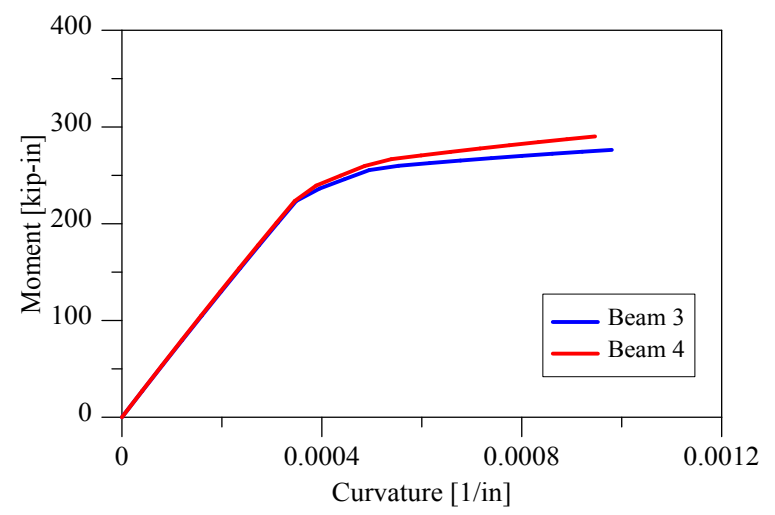

Figure 7. Moment-curvature relationship for Beams 3 and 4. $(1$ in. $=25.4$ $\mathrm{mm}, 1$ kip. $=4.45 \mathrm{kN}$ )

Experimental results are presented as plots of the total load applied (distributed equally to two point loads) and the displacement at midspan of the beam. Figures 8 and 9 experimentally show the impact of adding the PVC to the beams both as additional reinforcement and as repair following preliminary loading and damage. As expected, the PVC did not drastically impact the elastic behavior of the beams. In fact, all but one of the six tests had almost identical stiffness in the elastic range. Only one specimen, Beam 1R, deviated from this trend. The impact is fairly minor however due to the fact that the specimens still yielded at the same displacement. While the PVC did not have a major impact on the elastic behavior of the beam, its effect on the post-elastic behavior was noticeable. In each 
case the PVC provided a $10-15 \%$ increase in strength, a fact which is consistent with the expected results. Specifically, Beam 2 yielded at the same point (load and displacement) as Beam 1 but increased in strength compared to Beam 1 at a linear rate post-yield. Conversely, Beam 4 yielded at a higher load and displacement than did Beam 3, but maintained a consistent increase in strength in the post-yield region.

The repaired beams showed an even further increase in strength over those with pre-installed PVC. Specifically, the strength of Beam 1R was approximately $30 \%$ higher than that of Beam 1; and Beam 3R was approximately $35 \%$ higher in strength than Beam 3. This is likely a result of the increased steel stress due to strain hardening in the steel following initial loading. This is expected, as strain hardening in the steel reinforcement can be expected to contribute approximately $15-20 \%$ to the strength of a beam loaded beyond the yield point, unloaded, and subsequently reloaded. This increase in addition to the $10-15 \%$ from the PVC material can be said to cause the overall 30\%-35\% increase in strength from the original unrepaired beam.

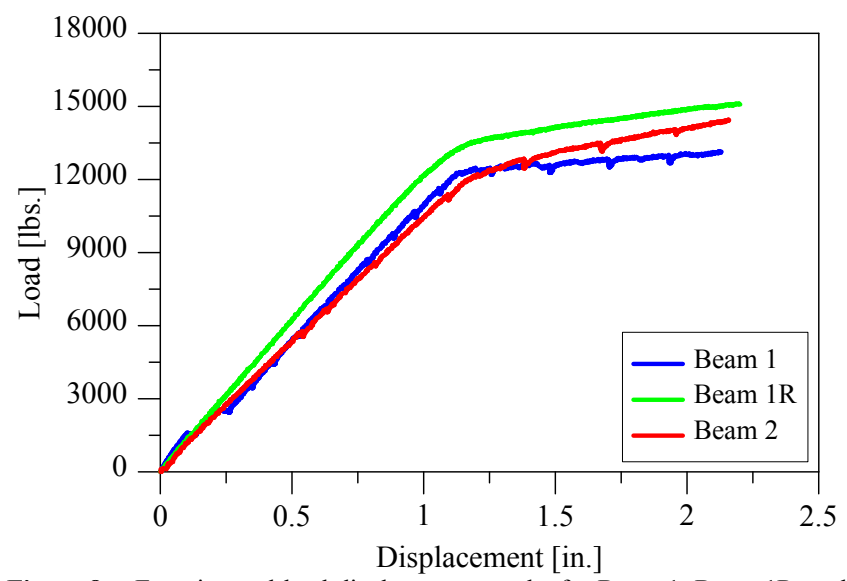

Figure 8. Experimental load-displacement results for Beam 1, Beam 1R, and Beam 2. $(1 \mathrm{in} .=25.4 \mathrm{~mm}, 1 \mathrm{lb} .=4.45 \mathrm{~N})$

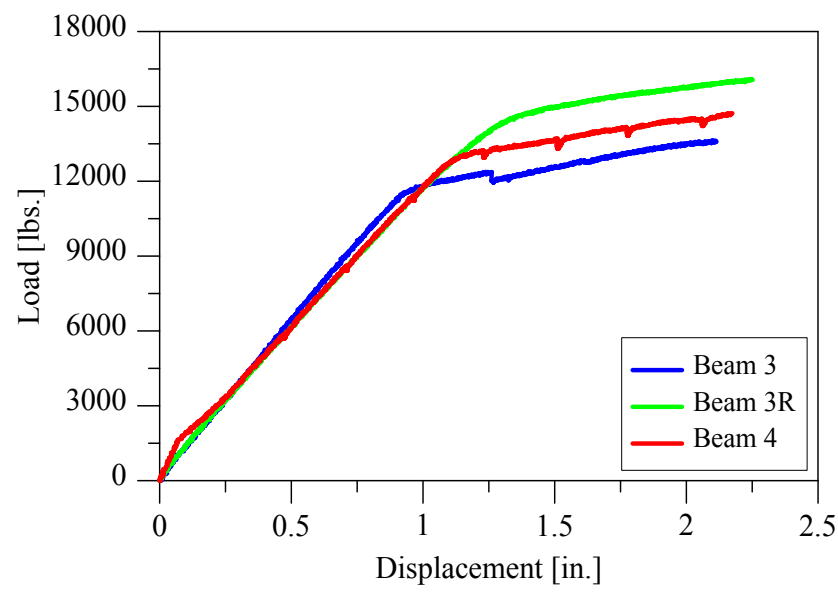

Figure 9. Experimental load-displacement results for Beam 3, Beam 3R, and Beam 4. $(1 \mathrm{in} .=25.4 \mathrm{~mm}, 1 \mathrm{lb} .=4.45 \mathrm{~N})$

Damage and cracking patterns were very similar for all test specimens. Some forms of repair aim to reduce the physical damage to the beam (cracking, spalling, etc.). Because the PVC has a much lower stiffness than the reinforced concrete, mitigating cracking was not a major objective. Due to the screws being anchored in the cover concrete, additional damage was observed at several of the screw locations at high displacement levels. Specifically additional cracks initiated at the screw locations. This cracking did not affect the behavior of the test specimens, nor did it induce spalling of the cover concrete, even at very high displacement levels. But it is indicative of one problem with this repair technique in that it does in fact introduce some damage to the beam. This is important to note, as this additional damage is a detriment to the overall improvement in behavior due to the additional material. Therefore, one key recommendation is that the screws be anchored in the confined portion of the concrete in full-scale applications to help mitigate the damage of the anchors.

\section{Conclusions}

A total of six tests were conducted on four specimens to assess the impact of using PVC angles as a simple and modest repair technique for reinforced concrete beams. The main conclusions of the study are summarized below. Please note that these conclusions are based on the small scale tests performed. Therefore caution should be exercised when extrapolating these results to full scale beams.

- The PVC angles provided a $10-15 \%$ increase in strength in the tested specimens. That increase is consistent with the expected increase in flexural strength due to the addition of PVC material. While the increase in strength is not substantial, it is sufficient for cases where only minor repair is needed. Therefore, it can be considered a cost-effective alternative to more expensive repair techniques that aim to dramatically change the behavior.

- Due to the relatively low stiffness of the PVC material, the elastic behavior of the beams is unaffected by the application of repair. This fact means that the elastic behavior of the structure as a whole is not going to change. This is an important fact in repair/retrofit considerations as changes to the elastic properties can have effects on the overall system behavior.

- Because the PVC angles are attached via concrete screws and not epoxy resin, delamination failure is not possible. Rather, the only failure that might be expected in the PVC is a bearing failure at the connection points. However, due to the flexibility of the material, this failure mode is unlikely.

When attaching the PVC angles, it is important to embed the anchor screws sufficiently into the beam core. This will ensure that shear is adequately transferred between the concrete and PVC even if the cover concrete begins to spall at high displacement 
levels.

\section{Acknowledgements}

The authors acknowledge the Department of Civil Engineering at California State University Fullerton for support of this research.

\section{REFERENCES}

[1] A. Balsamo, A. Colombo, G. Manfredi, P. Negro, A. Prota. Seismic behavior of a full-scale RC frame repaired using CFRP laminates.Engineering Structures, Volume 27, Issue 5, (2005): 769-780.

[2] M. Murra. Epoxy injection welds cracks back together. Concrete Construction 3 (1987).

[3] D. Fowler. Polymers in concrete: A vision for the $21^{\text {st }}$ century. Cement and Concrete Composites, Volume 21, Issues 5-6, (2006): 449-452.
[4] G. Chynoweth, et al. Concrete repair guide. ACI committee, concrete repair manual 546 (1996): 287-327.

[5] N. Grace. Concrete repair with CFRP. Concrete international 26.5 (2004): 45-52.

[6] S. Pendhari, T. Kant, Y. Desai. Application of polymer composites in civil construction: A general review. Composite Structures 84 (2008): 114-124.

[7] P. R. Chakrabarti, D. Millar, and S. Bandyopadhyay. Application of composites in infrastructure. Proceedings of the Third International Conference on Composites in Infrastructure(2002).

[8] C. Leung. Delamination failure in concrete beams retrofitted with a bonded plate. Journal of Materials in Civil Engineering 13 (2001): 106-113.

[9] D. Naish. Testing and modeling of reinforced concrete coupling beams. Dissertation. University of California Los Angeles (2010): $251 \mathrm{pp}$.

[10] E. Hognestad, N. W. Hanson, and D. McHenry. Concrete stress distribution in ultimate strength design.ACI Journal Proceedings. Vol. 52. No. 12. (1955) 